\title{
CORRECTION
}

\section{Correction to: Thermometry and interpretation of body temperature}

\section{Wenxi Chen ${ }^{1}$}

Published online: 25 February 2019

(c) Korean Society of Medical and Biological Engineering 2019

\section{Correction to: Biomedical Engineering Letters} https://doi.org/10.1007/s13534-019-00102-2

The author would like to add "@Togawa T." in the Figure 1 caption for the online published article.

Figure 1 caption should read as

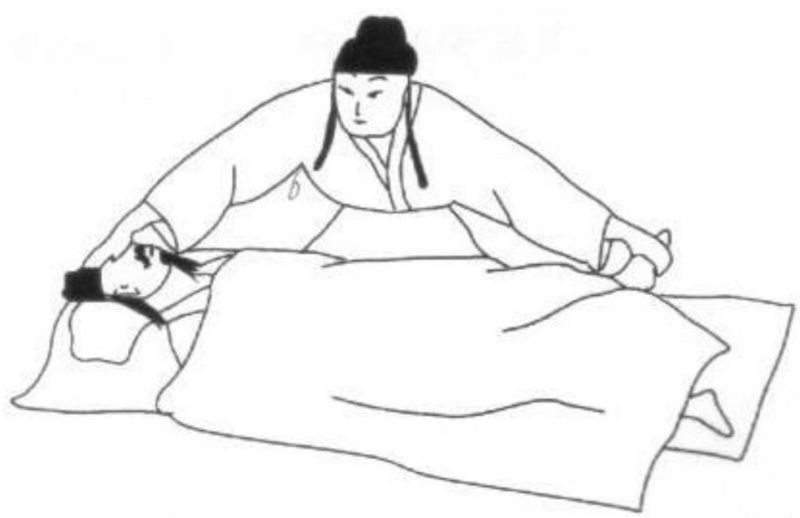

Fig. 1 Archaic method for diagnosis of disease using the body temperature difference between head and foot by touching a subject (O)Togawa T

Publisher's Note Springer Nature remains neutral with regard to jurisdictional claims in published maps and institutional affiliations.

The original article can be found online at https://doi.org/10.1007/ s13534-019-00102-2.

Wenxi Chen

wenxi@u-aizu.ac.jp

1 Biomedical Information Technology Laboratory, Research Center for Advanced Information Science and Technology, The University of Aizu, Aizu-Wakamatsu, Fukushima, Japan 